\title{
Numerical Simulation of Hot Gas Dispersion from a Chimney*
}

\author{
Daniel FUENTES DEL RÍO** and Takeo KAJISHIMA** \\ ** Department of Mechanical Engineering, Osaka University \\ 2-1 Yamada-oka, Suita, Osaka, 565-0871, Japan \\ E-mail: daniel@fluid.mech.eng.osaka-u.ac.jp
}

\begin{abstract}
This paper reports on numerical simulations of the flow of hot gases released vertically from a chimney into a crosswind with velocity and temperature ratio corresponding to an experiment. The use of a weak compressible scheme, which is capable of taking into account thermal expansion at low Mach number is proposed in order to improve the simulation of the flow. Simulations with 3 different temperature ratios were performed using the proposed method and the incompressible scheme with the Boussinesq approximation. For the isothermal case, both schemes predicted similar trajectories and had good agreement with experimental data. For high temperature ratios, our method showed better agreement with experimental data than the simulations with the method based on the Boussinesq approximation. It was found that the density changes due to thermal expansion near the nozzle exit act against buoyancy, diminishing the vertical velocity over the chimney affecting the trajectory of the flow, also modifying the areas where buoyancy has a considerable effect.
\end{abstract}

Key words : Jet, Turbulent Flow, Buoyancy, Boussinesq Approximation

\section{Introduction}

The release of exhausted gases from chimneys has a direct impact in the air quality of the surrounding areas downstream the source. Therefore, the understanding and accurate prediction of the dispersion of released gases in the atmosphere is essential in order to measure or predict environmental impact and in the improvement of actual designs.

The flow of gases from a chimney is highly complex and very often turbulent. Besides, the same kind of flow has many other industrial and environmental applications, like the dispersion of gases from erupting volcanoes, thermal discharges in oceans and rivers, fuel injection of burners and cooling of electronic devices. An extensive number of numerical and experimental works have been performed aiming different applications. In this paper, only a few representative works are cited. Margason ${ }^{(1)}$ summarized the experimental and numerical research performed on jets in crossflow until the 1990's dividing it in 3 main stages. Besides Margason classification, previous reported research can be divided in works with jet exit at the wall level and works with the jet exit in an elevated position, considering a chimney. Most of the experimental and numerical work focused on jets released from the bottom wall.

The first experimental works focused on the visualization of the flow and study the trajectory of the plume as function of the flow parameters, like plume to crosswind velocity ratio and Reynolds number, measuring velocity distributions for applications that ranged from jets in crossflow to V/STOL aircraft. Keffer and Baines ${ }^{(2)}$ used the hot wire anemometry technique and gave a general description of the development of the flow with variations of the velocity ratio. Kamotani and Greber ${ }^{(3)}$ considered temperature differences between the plume and crosswind describing the temperature distribution of the flow. Later, with the development of measuring techniques like hot-wire or laser-Doppler and with the improvement of visualization techniques, the experimental works focused on measuring the mean and fluctuating 
velocities, collecting turbulence statistics, determining the structure of the flow and identifying the formation of coherent turbulent structures, as well as turbulent mixing and dispersion studies. One of the most representative experimental works was performed by Andreopoulos and Rodi ${ }^{(4)}$. They used triple hot wire proves for the measurement of the instantaneous velocity field, describing the mean flow and turbulence field. During the 1990's, many works focused on the study of the wake downstream the jet exit. The most representative were performed by Fric and Roshko ${ }^{(5)}$ for the wall-jet case, and Eiff and $\mathrm{Keffer}^{(6)}$ for a jet released from an elevated position. Recently with the use of Particle Image Velocimetry (PIV), experimental results which involve temperature difference between the plume and the crossflow have been performed. In this area, Tezuka et al. ${ }^{(7)}$ conducted a series of experiments on a jet released from a stack considering different jet temperatures. They also performed a numerical simulation and made an estimation of the temperature field.

Previous experimental works concluded that the flow of gases released into a crosswind can be divided in three main regions. In the first region, the jet interacts with the crosswind, developing a shear layer near the exit. In the second region, the jet experiences maximum deflection, the formation of a mixing layer as well as transition to fully developed turbulence. In the third region, the direction of the jet becomes practically parallel to the crosswind, the velocity of the jet becomes similar to the crossflow and the jet practically mixes with it. In these three regions, the formation of turbulent structures has been observed. Fric and Roshko ${ }^{(5)}$ described four types of turbulent structures, shear layer vortices, horseshoe vortices, counterrotating vortex pair (CVP) and wake vortices. In case of a gas released from an elevated position, the wake vortices are produced by the stack and the jet itself. As for the shape of the jet exit, although most of the numerical and experimental work have been performed on round jets, Pathak et al. ${ }^{(8)}$ reported experimentally and numerically that the pressure profiles, penetration and trajectory is similar on round and square jets.

In terms of numerical work, many calculations aiming very different applications, that started from 2D prediction of gas dispersion, film blade cooling for gas turbines, combustion, to atmospheric contaminant dispersion. One of the most representative numerical works was performed by Yuan et al. ${ }^{(9)}$. They used LES for calculating the isothermal case for various velocity ratios. Yuan et al. ${ }^{(9)}$ made a description of the near field flow, turbulence dynamics and the development of turbulent coherent structures. Sau et al. ${ }^{(10)}$ analyzed the flow and vorticity dynamics of a square jet. Later, Said et al. ${ }^{(11)}$ conducted an experimental and numerical work focusing on the pollutant dispersion released from a smokestack.

The development of numerical techniques allowed to have detailed descriptions of the structure of the flow. The low Mach number of the flow makes suitable the use of the algorithm for incompressible flow. Therefore, the majority of the previously reported numerical simulations were performed in isothermal conditions using incompressible schemes, obtaining good agreement with experimental data. However, in most environmental and in some industrial applications, there is a broad temperature difference between the the plume and the surrounding atmosphere, that produces significant buoyant forces and density variations, modifying the trajectory of the plume.

Numerical simulations of flows with non-isothermal conditions have been also performed. In order to take into account the effects of buoyancy, in some works like Tezuka et al. ${ }^{(7)}$, the incompressible scheme with the Boussinesq approximation for buoyancy treatment was used. However, disagreement with experimental data was observed. This disagreement has two main reasons. First, the incompressible scheme does not take into account density variations that arise in the flow. Second, the conditions of velocity ratio and blowing ratio cannot be satisfied at the same time when density is considered constant.

In order to take into account the effect of buoyancy and density changes due to broad temperature differences, the use of the weak compressibility method is appropriate for low Mach number flow.

This paper reports on numerical simulations of the flow of hot gases from a chimney. A 
warm jet is released perpendicularly from an elevated position into a crosswind and mixes with it. Simulations were performed at 3.3 jet to crosswind velocity ratio, with crosswind temperature of $300 \mathrm{~K}$, and three different plume temperatures. In order compare and understand the influence of thermal expansion and small compressibility, simulations using an incompressible scheme with the Boussinesq approximation, and simulations using a weak compressible scheme were performed. The results obtained with both schemes, are compared with experimental data for validation.

\section{Conservation Equations}

\subsection{Incompressible scheme}

For the incompressible scheme, the dimensionless continuity, momentum and energy equations are considered. The Boussinesq approximation, used for buoyancy treatment, assumes that the density is constant in all terms except for the body force term in the momentum equation. Then, buoyancy is approximated by the body force term in the momentum equation. These equations are expressed as follows:

$$
\begin{aligned}
& \frac{\partial u_{i}}{\partial x_{i}}=0 \\
& \rho\left(\frac{\partial u_{i}}{\partial t}+\frac{\partial u_{i} u_{j}}{\partial x_{j}}\right)=-\frac{\partial p}{\partial x_{i}}+\frac{1}{\operatorname{Re}} \frac{\partial^{2} u_{i}}{\partial x_{j} \partial x_{j}}-\frac{G r}{\operatorname{Re}^{2}} T^{\prime} g_{i}, \\
& \frac{\partial T}{\partial t}+\frac{\partial u_{i} T}{\partial x_{i}}=\frac{1}{\operatorname{RePr}} \frac{\partial^{2} T}{\partial x_{i} \partial x_{i}} .
\end{aligned}
$$

The velocity $u_{i}$ is nondimensionalized with the initial jet velocity $V_{j e t}, u_{i}=u_{i}^{*} / V_{j e t}$, the density $\rho$ with the crosswind initial density $\rho_{0}, \rho=\rho^{*} / \rho_{0}$, and the temperature $T$ with the crosswind initial temperature $T_{0}, T=T^{*} / T_{0}$, and $T^{\prime}=\left(T^{*}-T_{0}\right) /\left(T_{\text {jet }}-T_{0}\right)$, where $T_{j e t}$ is the plume initial temperature and $g_{i}=-j$ is the dimensionless gravity vector. The representative length scale $l$ is the inner diameter of the chimney. The superindex * indicates that the variable is dimensional. The pressure $p$ is defined as $p=\left(P^{*}-P_{0}\right) /\left(\rho_{0} V_{j e t}^{2}\right)$, where $P_{0}$ is the initial pressure. The relevant dimensionless parameters are: Reynolds number, $R e=\rho_{0} V_{j e t} l / \mu_{0}$, the Grashof number $G r=g \beta \rho_{0}^{2} l^{3}\left(T_{j e t}-T_{0}\right) / \mu_{0}^{2}$, and the Prandtl number, $\operatorname{Pr}=v_{0} / \alpha_{0}$, where $\beta$ is the coefficient of thermal expansion. Viscosity $\mu_{0}$, kinematic viscosity $v_{0}$ and thermal diffusivity $\alpha_{0}$ are assumed to be constant.

\subsection{Compressible scheme}

The full compressible continuity, momentum and energy equations are:

$$
\begin{aligned}
& \frac{\partial \rho}{\partial t}+\frac{\partial \rho u_{i}}{\partial x_{i}}=0 \\
& \frac{\partial \rho u_{i}}{\partial t}+\frac{\partial \rho u_{i} u_{j}}{\partial x_{j}}=-\frac{1}{\gamma M a^{2}} \frac{\partial p}{\partial x_{i}}-\frac{1}{R e} \frac{\partial \sigma_{i j}}{\partial x_{j}}-\frac{G r}{R e^{2}} T^{\prime} g_{i} \\
& \frac{\partial \rho T}{\partial t}+\frac{\partial \rho u_{j} T}{\partial x_{j}}=\frac{\gamma-1}{\gamma}\left(\frac{\partial p}{\partial t}+u_{j} \frac{\partial p}{\partial x_{j}}\right)+(\gamma-1) \frac{M a^{2}}{R e} \sigma_{i j} \frac{\partial u_{i}}{\partial x_{j}}-\frac{1}{R e P r} \frac{\partial^{2} T}{\partial x_{i} \partial x_{i}}, \text { (6) }
\end{aligned}
$$

where $\gamma$ is specific heats ratio and $\sigma_{i j}$ the viscous stress:

$$
\sigma_{i j}=-\mu\left(\frac{\partial u_{i}}{\partial x_{j}}+\frac{\partial u_{j}}{\partial x_{i}}-\frac{2}{3} \delta_{i j} \frac{\partial u_{k}}{\partial x_{k}}\right)
$$

where $\mu$ is viscosity considered as $\mu=\mu^{*} / \mu_{0}$, and $\delta_{i j}$ is the Kronecker delta. The ideal gas is assumed and the equation of state is given in a dimensionless way as:

$$
p=\rho T \text {. }
$$

The length scale $l$ and the nondimensionalization parameters for velocity $u_{i}$, temperature $T$, density $\rho$ are the same as the incompressible scheme. For the compressible scheme, the pressure $p$ is $p=p^{*} / \rho_{0} R T_{0}$. The relevant dimensionless parameters are the same as the incompressible scheme, with the addition of the Mach number, $M a=V_{j e t} / \sqrt{\gamma R T_{0}}$. 


\section{Numerical procedure}

\subsection{Flow configuration}

As a basically similar configuration with the experimental setup of Tezuka et al. ${ }^{(7)}$ in an open duct, the computational domain geometry and size is shown in Figure 1. Uniform flow is given for the inflow boundary condition of the crosswind and the flow from the chimney. The two lateral, top and bottom walls are assumed adiabatic free-slip walls with gradientfree pressure. The inner and outer diameter of the chimney is assumed to be the same. The temperature of the ambient crosswind inflow is constant at $T_{0}=300 \mathrm{~K}$, while simulations with three different temperatures for the jet inflow $T_{j}, 300 \mathrm{~K}, 343 \mathrm{~K}$ and $393 \mathrm{~K}$ were performed. The Reynolds number is $R e=2000$, which is the same as in the experiment ${ }^{(7)}$. The Prandtl number is $\operatorname{Pr}=0.7$. The jet to crossflow relations are resumed in Table 1 .

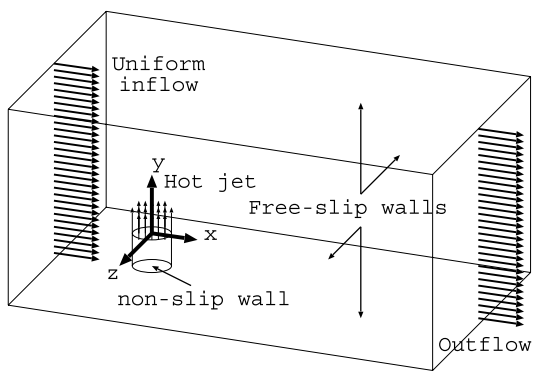

(a) Geometry and boundary conditions

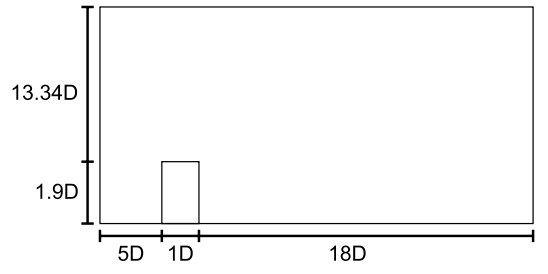

(b) Domain size in $\mathrm{x}-\mathrm{y}$ cross-section.

Fig. 1

The convective outflow boundary condition is given at the outflow cross-section:

$$
\frac{\partial u_{i}}{\partial t}+u_{m} \frac{\partial u_{i}}{\partial x}=0
$$

where $u_{m}$ is an average velocity at the outlet cross-section.

The coordinate system is also shown in Figure 1, $x$ represents the distance in the streamwise direction, $y$ in the vertical direction, and $z$ in the span-wise direction, and the origin is located at the center of the chimney exit. Table 2 shows the length of the domain given in stack diameters in each direction, the total number of grid points and the number of grid points per diameter in each direction. The distribution of grid points is uniform.

A formal grid refinement study was not performed in this work. However, the numerical results showed good agreement with experimental data at the corresponding Reynolds number. Other authors using LES for simulating this kind of flows ${ }^{(9)(12)}$ reported that reasonable quantitative results are possible when the large-scale flow features are resolved. The mesh used in this research is fine enough to resolve the large-scale characteristics of the flow, like the counter-rotating vortex pair. Also, the boundary surfaces of the domain are specified as free-slip boundaries, what eliminates the need of simulating the crosswind boundary layer, making the resolution requirements much less restrictive.

Table 1 Simulation flow conditions

\begin{tabular}{|cc|c|c|c|}
\hline \multicolumn{2}{|c|}{ Case } & I & II & III \\
\hline $\begin{array}{c}\text { Temperature } \\
\text { ratio }\end{array}$ & $\frac{T_{\text {jet }}}{T_{0}}$ & 1.0 & 1.14 & 1.31 \\
\hline $\begin{array}{c}\text { Velocity } \\
\text { ratio }\end{array}$ & $\frac{V_{\text {jet }}}{u_{0}}$ & 3.3 & 3.3 & 3.3 \\
\hline $\begin{array}{c}\text { Blowing } \\
\text { ratio }\end{array}$ & {$\left[\frac{\left(\rho u^{2}\right)_{j e t}}{\left(\rho u^{2}\right)_{0}}\right]^{1 / 2}$} & 3.33 & 3.12 & 2.91 \\
\hline
\end{tabular}


Table 2 Domain size in stack diameters and number of cells.

\begin{tabular}{|c|c|c|c|}
\hline Direction & Length & Cells & Cells/diameter \\
\hline $\mathrm{x}$ & 24 & 600 & 25 \\
\hline $\mathrm{y}$ & 15.24 & 320 & 21 \\
\hline $\mathrm{z}$ & 9.23 & 240 & 26 \\
\hline
\end{tabular}

\subsection{Numerical method}

In the solution of the basic equations for the incompressible fluid flow Eqs. (1), (2) and (3), and of the weak compressible fluid flow Eqs. (4), (5) and (6), the fractional-step method is applied. The second order central finite difference scheme is used for spatial discretization. For temporal discretization, the second order Adams-Bashforth method is used in the convective, viscous and body force terms, while the Backward Euler method is used for the pressure and continuity equations.

3.2.1. A non-Boussinesq scheme As expressed in section 1, in the majority of cases of the flow released from a chimney, high temperature differences and very low Mach number are involved. Since the Mach number is very low, around $M a=3 \times 10^{-3}$ for the experimental conditions of Tezuka et al. ${ }^{(7)}$, the flow is usually treated as incompressible, however, since there are considerable temperature differences, thermal expansion and small compressibility should be taken into account. The difference of pressure in a time increment $\Delta t$ can be expressed as:

$$
\Delta P=\left(\frac{\partial p}{\partial \rho}\right)_{T} \Delta \rho+\left(\frac{\partial p}{\partial T}\right)_{\rho} \Delta T .
$$

Equation (10) can be written non-dimensionally as:

$$
\Delta p=T \Delta \rho+\rho \Delta T
$$

where $\Delta \phi=\phi^{n+1}-\phi^{n}$, and $n$ denotes the time-step count. Now, the implicit form of the continuity equation 4 can be written as:

$$
\Delta \rho=-\frac{\partial}{\partial x_{i}}\left(\rho u_{i}\right)^{n+1} \Delta t
$$

In a similar form to the fractional-step method for incompressible flows, the momentum conservation can be expressed as:

$$
\left(\rho u_{i}\right)^{F}=\left(\rho u_{i}\right)^{n}+\Delta t\left(\frac{3 A_{i}^{n}-A_{i}^{n-1}}{2}\right)
$$

and

$$
\left(\rho u_{i}\right)^{n+1}=\left(\rho u_{i}\right)^{F}-\frac{\Delta t}{\gamma M a^{2}} \frac{\partial p^{n+1}}{\partial x_{i}}
$$

where $A_{i}$ includes the convective, viscous and body force terms of the momentum conservation equation. Particularly, the highly conservative scheme ${ }^{(13)}$ is applied to the convective term. However, the order of accuracy and the variable arrangement are not the same as in the former work $^{(13)}$.

Now, the divergence of Eq. (14) derives

$$
-\frac{1}{\gamma M a^{2}} \frac{\partial^{2} p^{n+1}}{\partial x_{i} \partial x_{i}}=\frac{1}{\Delta t} \frac{\partial\left(\rho u_{i}\right)^{n+1}}{\partial x_{i}}-\frac{1}{\Delta t} \frac{\partial\left(\rho u_{i}\right)^{F}}{\partial x_{i}} \text {. }
$$

Obtaining $T^{n+1}$ from Eq. (6) and assuming $\Delta p=T^{n} \Delta \rho+\rho^{n} \Delta T$ for Eq. (11), Eq. (12) becomes

$$
\frac{\partial}{\partial x_{i}}\left(\rho u_{i}\right)^{n+1}=\frac{\Delta p-\rho^{n} \Delta T}{T^{n} \Delta t} .
$$

Then, the following equation can be obtained.

$$
\frac{1}{\gamma M a^{2}} \frac{\partial^{2} p^{n+1}}{\partial x_{i} \partial x_{i}}-\frac{1}{T^{n}(\Delta t)^{2}} p^{n+1}=\frac{-p^{n}-\rho^{n}\left(T^{n+1}-T^{n}\right)}{T^{n}(\Delta t)^{2}}+\frac{1}{\Delta t} \frac{\partial\left(\rho u_{i}\right)^{F}}{\partial x_{i}} .
$$

This equation is solved using the Jacobi method. Then, we obtain $\left(\rho u_{i}\right)^{n+1}$ by Eq. (14), and $\rho^{n+1}$ by Eq. (4). 


\section{Results and Discussion}

\subsection{Trajectory}

The release of hot gases from an elevated position generates a complicated flow and interaction between the gases and the crosswind. In the present work as in the experimental data $^{(7)}$, the trajectory of the flow is defined by the streamline originating from the center of the chimney exit. Figure 2 shows a comparison of the jet axis trajectory between experimental and numerical results, using the Boussinesq and the non-Boussinesq schemes for 3 different temperature ratios. The hot gas inflow, originally in vertical direction, interacts with the crossflow and bends into the horizontal direction a few diameters downstream, however it does not become completely horizontal.

Both schemes calculate similar jet trajectories and show good agreement with experimen-
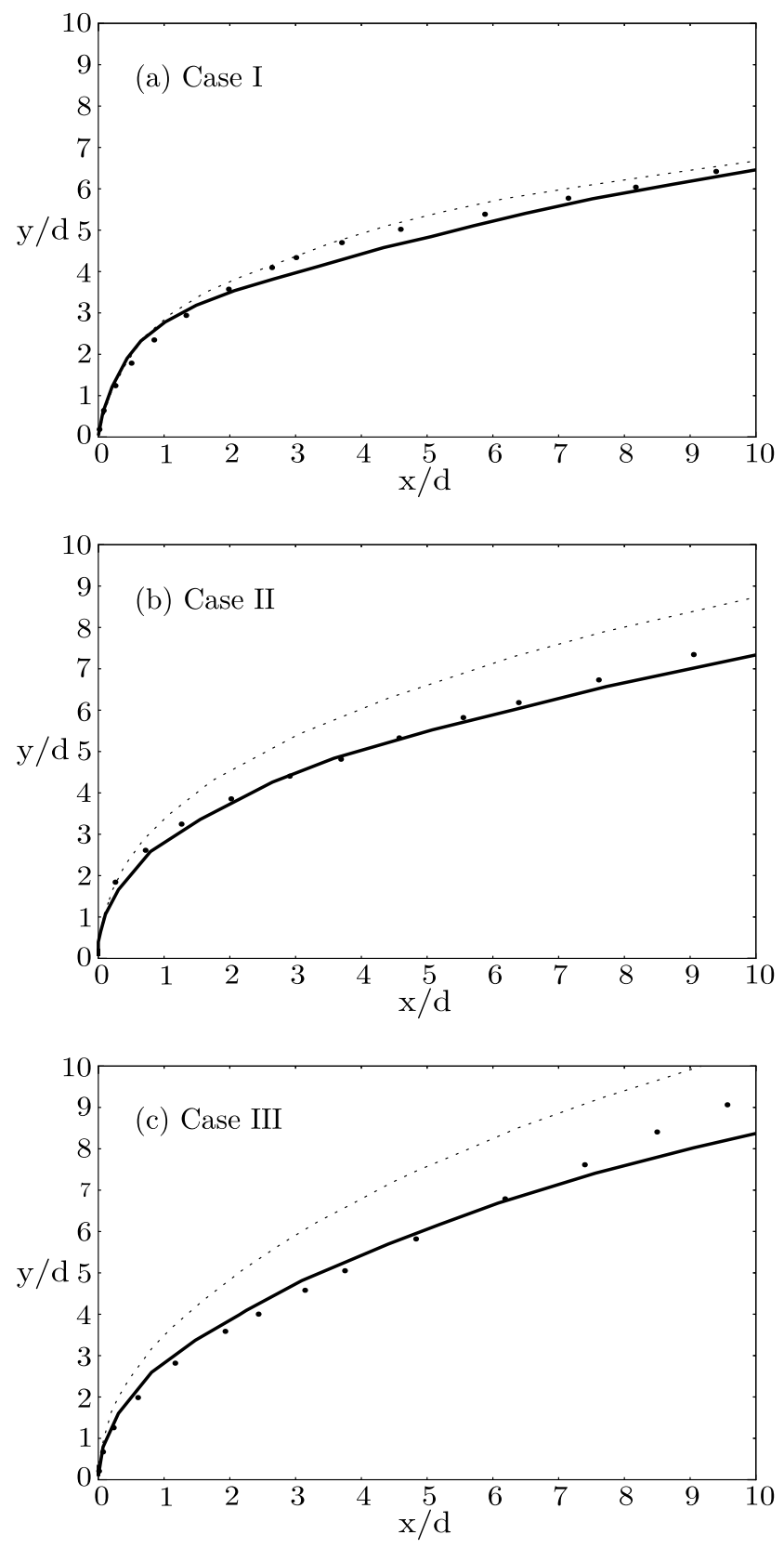

Fig. 2 Comparison of the jet trajectory. $(\bullet)$ experimental,(...) Boussinesq and (-) nonBoussinesq. 
tal data in case I. In cases II and III, the weak compressible scheme shows better agreement with experimental data. It is observed that in both cases, the Boussinesq approximation calculate higher trajectory than the experimental data and the weak compressible scheme. This has two main reasons. First, as shown in Table 1, there is an inevitable difference in the conditions of the simulation. Since the density is considered constant in the incompressible scheme, the blowing ratio also remains constant even when the jet temperature changes. Second, from 1 to 3 diameters downstream the jet exit, fast temperature changes are expected, what enhances thermal expansion and affect the trajectory of the flow.

\subsection{Mean velocity field}

Figure 3 is a plot of the time-averaged velocity field for the non-Boussinesq scheme in an $\mathrm{x}-\mathrm{y}$ cross-section located at the center of the jet for case II. A fast velocity decrease is observed between the exit of the jet and 4 diameters downstream. From this point, the velocity magnitude becomes similar to the crossflow velocity, indicating strong interaction and mixing with the crossflow. The stack and the jet itself have a similar effect of a blunt body inside the flow, forming a wake-like flow downstream the stack and the jet. As a consequence, a reverse-flow in the lee side of the jet is observed. The formation of this reverse flow suggests that the entrainment of cool flow into the hot gases starts from the release of it.

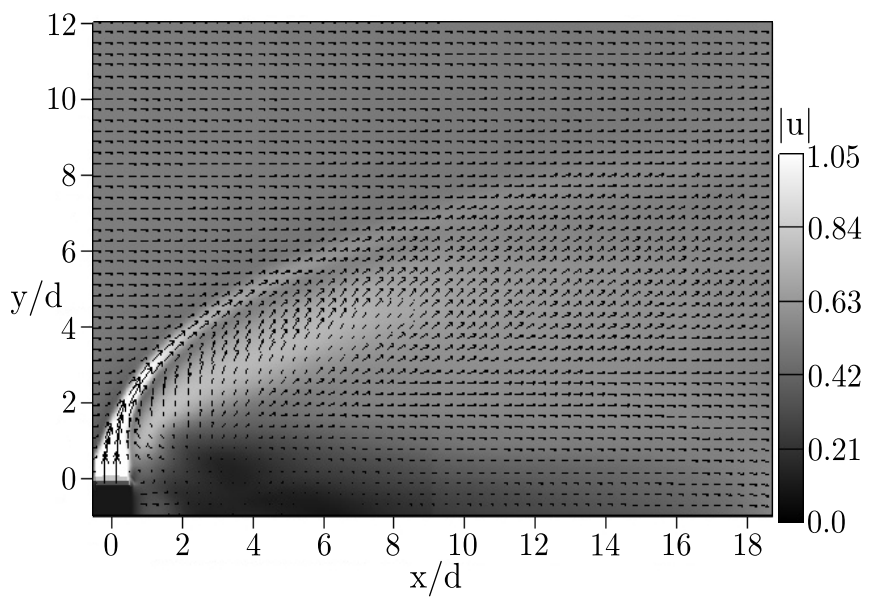

Fig. 3 Velocity vectors and magnitude of the mean velocity field for Case II in the cross-section $z=0$.

4.2.1. Horizontal velocity

Figures 4 compare the time averaged horizontal velocity $u$ calculated with both schemes over the center of the chimney $z=0$ at different positions downstream the stack. For all cases, from 1 to 2 diameters downstream the stack, the horizontal velocity is negative, this area corresponds to the reverse flow mentioned above. Said et al. ${ }^{(14)}$ suggested that the reverse flow depends on the Reynolds number and the velocity ratio. The incompressible scheme calculated a similar negative velocity for all cases, indicating that the magnitude of the flow is independent of the temperature ratio. However, the weak compressible scheme calculated different velocity for each case. Larger temperature ratios provoke stronger density gradients close to the exit of the jet, causing the increase in the reverse flow, indicating that there is also a dependency on the temperature ratio.

For all cases, the streamwise velocity maximum correspond to the horizontal velocity of the core of the jet. In case I, both schemes predict similar streamwise velocity profiles, a small difference in the velocity magnitude and elevation of the jet core is noticed from 3 diameters downstream. In cases II and III, both schemes predict similar streamwise velocity magnitude. However, an important difference in the position of the velocity maximum is noted. 


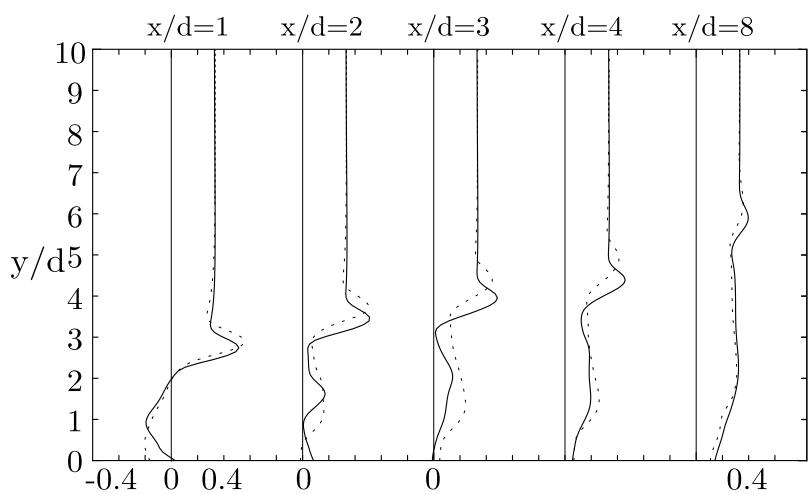

(a) Case I

$\mathrm{U} / \mathrm{Vj}$

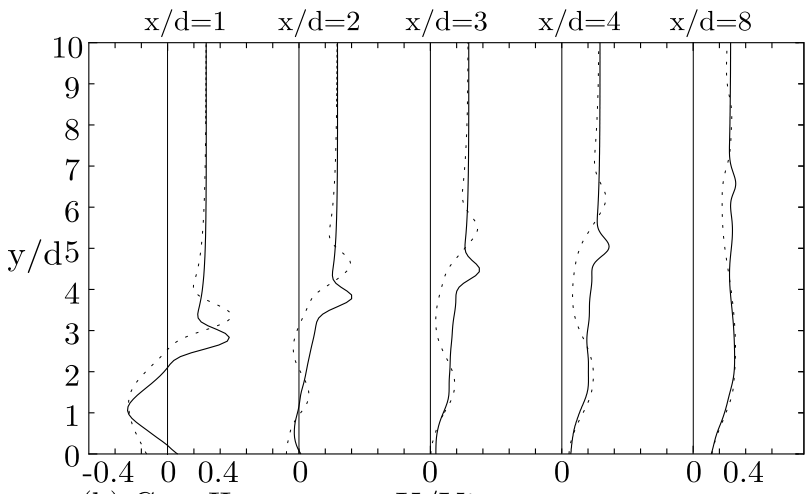

(b) Case II

$\mathrm{U} / \mathrm{Vj}$

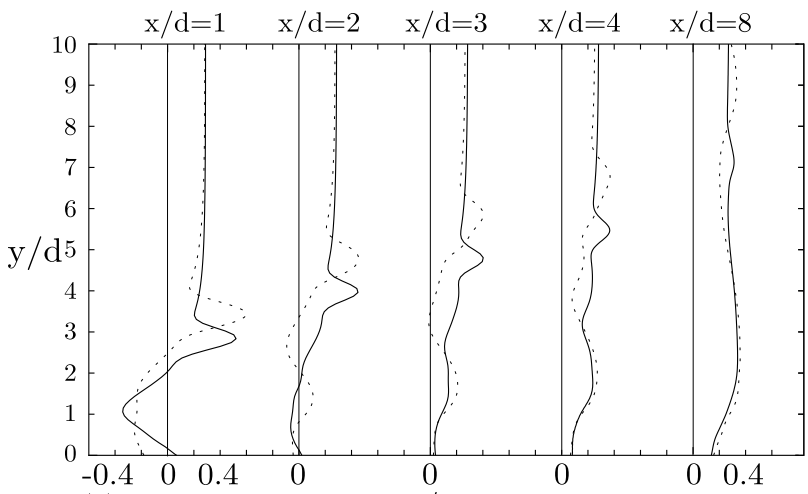

(c) Case III

$\mathrm{U} / \mathrm{Vj}$

Fig. 4 Mean horizontal velocity at the center of the jet.(…) Boussinesq and (-) nonBoussinesq.

4.2.2. Vertical velocity

Figures 5 show a comparison of vertical velocity predicted with both schemes in different positions downstream the stack at the center of the jet $z=0$ for the 3 cases. In all cases, from 1 diameter there are two different points where the vertical velocity is a maximum. The lower peak indicates the entrainment of cool fluid into the hot gases core, that is a consequence of the formation of a counter-rotating vortex pair (CVP) in the streamwise direction. The higher peak is provoked by the flow from the chimney. For all cases, a fast decrease in vertical velocity is observed in areas close to the stack.

In case I, where the effect of buoyancy is not present, the vertical velocity becomes almost half of the original just one diameter downstream the stack. Further downstream, both schemes predict similar vertical velocity magnitude, with small differences in the elevation of the jet core and in the vertical velocity peak due to the CVP.

In cases II and III an important difference in magnitude and location of the velocity maxima is noted, this difference widens with the temperature ratio. The effect of buoyancy makes the vertical velocity diminish at a smaller rate than case I, elevating the trajectory of the 


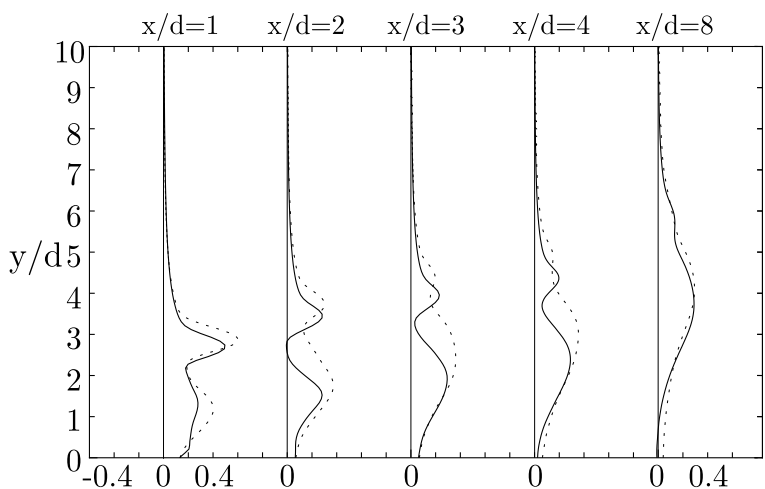

(a) Case I

$\mathrm{V} / \mathrm{Vj}$

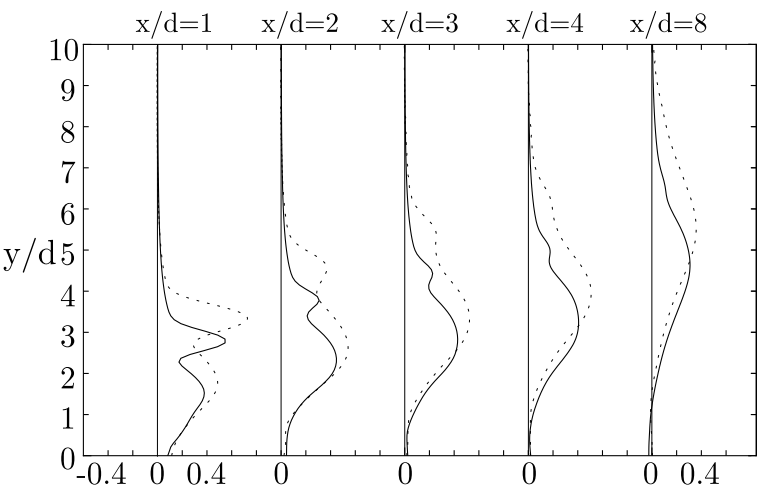

(b) Case II

$\mathrm{V} / \mathrm{Vj}$

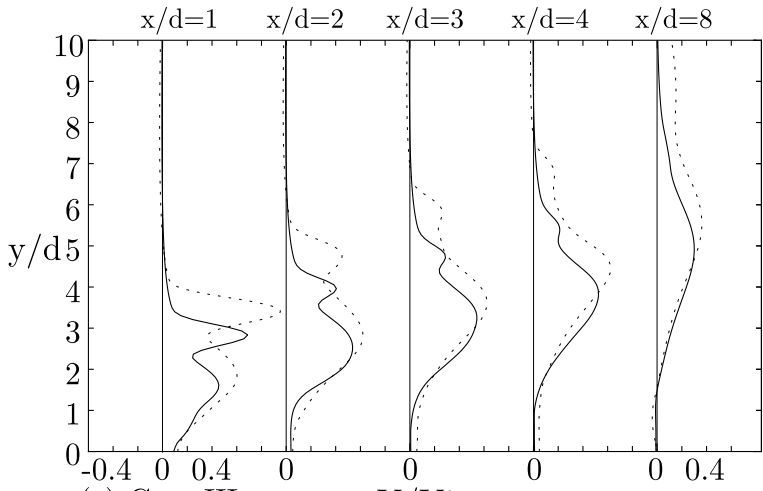

(c) Case III

$\mathrm{V} / \mathrm{Vj}$

Fig. 5 Mean vertical velocity at the center of the jet.(...) Boussinesq and (-) nonBoussinesq.

plume. Looking at Fig. 3, from 1 to 3 diameters downstream the stack, the jet bends into the direction of the crossflow and the velocity magnitude decreases the most. Fig. 5, shows the greatest disagreement of the vertical velocity calculated with both schemes in the same area. As will be pointed in section 4.3, a fast temperature decrease is observed in this area, what implies a fast density increase. The incompressible scheme only takes into account the effect of buoyancy. The weak compressible scheme also takes into account density changes. This suggests that thermal expansion acts against buoyancy, reducing the vertical velocity and as a consequence, the trajectory calculated with the weak compressible scheme becomes lower.

For all cases, the vertical velocity of the CVP center is maximum between 2 and 3 diameters downstream the stack. From this point, it start diminishing slowly, and becomes bigger than the vertical velocity of the jet. This indicates that the entrainment of cool air into the core of the jet continues downstream the bending of the jet, that the flow starts being dominated by the CVP and that this structure prevails further downstream than the core of the jet. 


\subsection{Mean temperature field}

Figure 6 shows time averaged temperature contours for the non-Boussinesq scheme in a $x-y$ cross-section along the center of the jet $z=0$. When the hot gases are released, the temperature of the fluid that is in touch with the crossflow decreases suddenly. In the lee side of the jet, density changes have an effect in the formation of the reverse flow mentioned in section 4.2. Also, a fast temperature decrease is observed in the bending of the jet. This suggest that the crossflow penetrated completely into the gas core. Also, this area coincides with the widest vertical velocity difference mentioned in section 4.2.2, indicating that density increase acts against buoyancy, changing the trajectory of the flow. From 4 diameters downstream the jet exit, the temperature decreases slowly, however, 18 diameters downstream the temperature of the hot gas core is still slightly higher than the crosswind.

Figure 7 shows z-y cross section of the time averaged temperature contours from both schemes at different positions downstream the stack for case III. From the hot gas release, the interaction of the hot gas with the crossflow and the entrainment of cool fluid into the jet core contribute to a change in the temperature distribution, from an original vertical oriented circle to an horizontal oriented kidney shape.

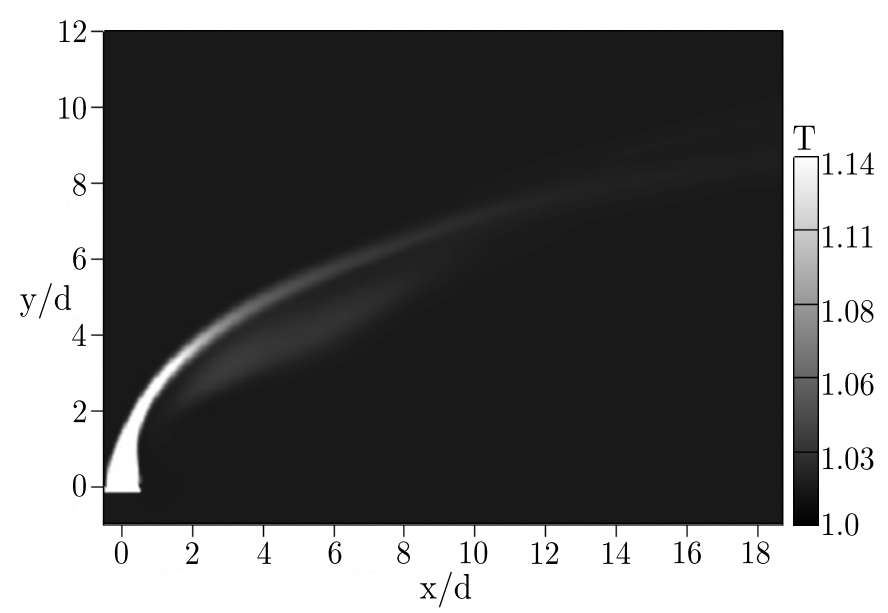

Fig. 6 Mean temperature field for case II in the cross-section $z=0$.

In case of the Boussinesq scheme, a higher vertical velocity provokes that the fluid has less time to transfer its heat to the crossflow and to mix with it. As a consequence, at $x / d=1$, where the temperature in the center of the jet is still high, both schemes calculate different maximum temperature. This suggest that for the Boussinesq scheme calculation, buoyancy has a considerable effect in wider areas, giving another reason for the discrepancy in the calculated trajectory. At $x / d=2$, the temperature of the hot gas core decreased considerably, indicating that at this point the effect of buoyancy reduces, at the same time, the temperature in the side areas of the kidney shape becomes similar than the center, indicating that the heat is being transferred to this areas. The warm side areas of the kidney shape, coincides with the center of each counter-rotating vortex.

Further downstream, this kidney shape starts expanding. From $x / d=3$, the temperature at the center of the jet decreases slowly, but at a faster rate than the kidney shape sides, what makes that from $x / d=4$ for the weak compressible scheme, and $x / d=6$ for the Boussinesq scheme, the temperature in the center of the jet becomes lower than the sides. From this points, the lower vertical velocity peak, Fig. 5, becomes clearly larger than the peak from the jet axis, suggesting that from $x / d=6$, the flow is dominated by the CVP. 

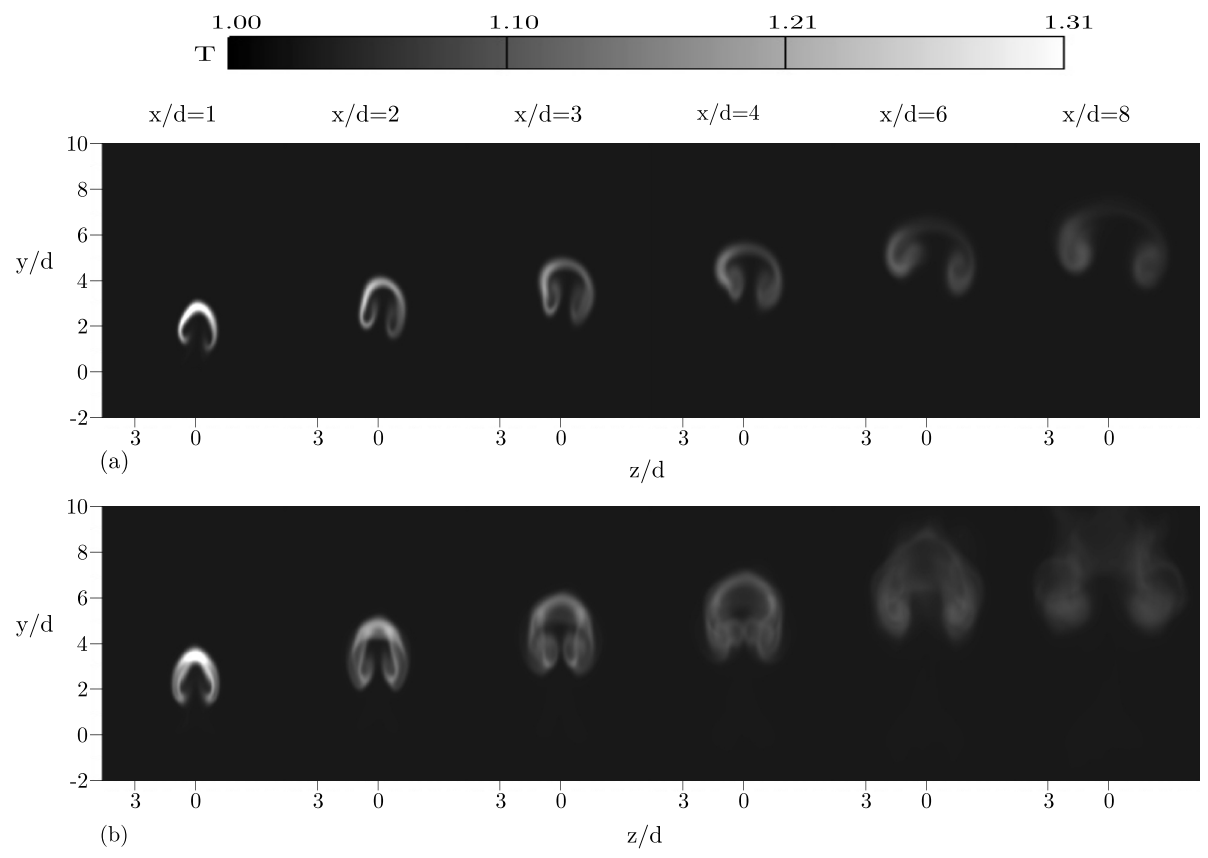

Fig. 7 Mean temperature contours in z-y cross-sections for Case III. a) nonBoussinesq. b) Boussinesq.

\section{Conclusions}

Numerical simulations of the release of a hot gas from a chimney into a crossflow were performed using the weak compressible scheme, and the Boussinesq approximation for buoyancy treatment. The results were compared with experimental data for three temperature ratios.

Qualitatively, both schemes reproduced the general characteristics of the flow. The trajectory calculated with both schemes showed good agreement with experimental data in the isothermal case. In cases with higher temperature ratios, the trajectory calculated with the weak compressible scheme showed better agreement with experimental data.

It was found that thermal expansion has an important effect in the development of the flow, specially in areas close to the chimney exit and in the bending of the jet. The fast temperature decrease in this area provokes density variations which induce a difference in the vertical velocity calculated with both schemes. Density increase acts against buoyancy slowing down the flow, making the weak compressible scheme calculate a lower trajectory than the incompressible scheme. The difference in the calculated vertical velocity also modifies the position and the maximum temperature of the bending of the jet, altering the areas where buoyancy has a strong effect.

Close to the chimney exit, the windward side of the jet is mainly affected by the shear flow between the jet and the crosswind. The lee side is influenced by the formation of a wake due to the presence of the stack and the jet itself, the formation of a counter-rotating vortex pair provoked by the entrainment of cool fluid into the jet core, and the formation of a reverse flow, which velocity depends on jet to cross-flow velocity ratio. The calculation with the weak compressible model showed that this reverse flow also increases with the temperature ratio, enhancing the entrainment of crosswind fluid into the plume, suggesting that the reverse flow also depends on the temperature ratio.

The temperature distribution changes from a vertical oriented circle to a kidney shape, which sides tend to separate. Downstream the bending of the jet, the areas of high temperature correspond to the center of each counter rotating vortex. The entrainment of cool air into the jet core make that the temperature inside the CVP become higher than the hot gas core, indicating that far downstream the exit of the jet, the flow is dominated by this structure. 


\section{References}

( 1 ) R. J. Margason. Fifty years of jet in cross flow research. In Computational and experimental assesment of jets in cross flow. AGARD, 1993.

( 2 ) J. F. Keffer and W. D. Baines. The round turbulent jet in a cross-wind. Journal of Fluid Mechanics, 15:481-496, 1963.

( 3 ) Y. Kamotani and I. Greber. Experiments on a turbulent jet in a cross flow. AIAA Journal, 10(11):1425-1429, 1972.

( 4 ) J. Andreopoulos and W. Rodi. Experimental investigacion of jets in a crossflow. Journal of Fluid Mechanics, 138:93-127, 1984.

( 5 ) T. F. Fric and A. Roshko. Vortical structure in the wake of a transverse jet. Journal of Fluid Mechanics, 279:1-47, 1994.

( 6 ) O. S. Eiff and J. F. Keffer. On the structures in the near-wake region of an elevated turbulent jet in a crossflow. Journal of Fluid Mechanics, 333:161-195, 1997.

( 7 ) H. Tezuka, M. Gomi, M. Mori, and K. Hishida. Piv measurement of a heated jet with cross flow and temperature estimacion by cfd. Transactions of the Japan Society of Mechanical Engineers, B, 75(757):1763-1773, 2009.

( 8 ) M. Pathak, A. Dewan, and A. K. Dass. Computational prediction of a slightly heated turbulent rectangular jet discharged into a narrow channel crossflow using two different turbulence models. International Journal of Heat and Mass Transfer, 49:3914-3928, 2006.

( 9 ) L. Yuan, R. L. Street, and J. H. Ferziger. Large-eddy simulations of a round jet in crossflow. Journal of Fluid Mechanics, 379:71-104, 1999.

(10) A. Sau, T. W. H. Sheu, S. F. Tsai, R. R. Hwang, and T. P. Chiang. Structural development of vortical flows around a square jet in cross-flow. Proceedings of the Royal Society of London A, 460:3339-3368, 2004.

(11) N. M. Said, H. Mhiri, G. Le Palec, and P. Bournot. Experimental and numerical analysis of pollutant dispersion from a chimney. Atmospheric Environment, 39:1727-1738, 2005.

(12) J.U. Schlüter and T. Schönfeld. Les of jets in cross flow and its application to a gas turbine burner. Flow, Turbulence and Combustion, 65:177-203, 2000.

(13) T. Kajishima, T. Ohta, K. Okazaki, and Y. Miyake. High-order finite difference method for incompressible flows using collocated grid system. JSME International Journal, 41:830-839, 1998.

(14) N. M. Said, S. Habli, H. Bournot, and G. Le Palec. Flow field measurement in a crossflowing elevated jet. Journal of Fluids Enginnering, 129:551-562, 2007. 